\title{
Ingestáo Oral e Grau de Comprometimento da Disfagia Orofaríngea Neurogênica Pré e Pós-Fonoterapia
}

\author{
Oral Intake and Severity Scale In Neurogenic Oropharyngeal Dysphagia
}

\section{Aline Rodrigues Pinto 1 , Paula Cristina Cola ${ }^{2}$ Lidia Raquel de Carvalho ${ }^{3}$, Suely Mayumi Motonagat, Roberta Gonçalves da Silva ${ }^{5}$}

\section{RESUMO}

Objetivo. Comparar o nível de ingestão oral e o grau de comprometimento da disfagia orofaríngea neurogênica pré e pós - fonoterapia. Método. 19 indivíduos com disfagia orofaríngea neurogênica, 10 adultos pós-Acidente Vascular Cerebral (AVC), faixa etária de 4 a 76 anos, que compunham o grupo 1 (G1) e nove crianças com Encefalopatia Crônica Infantil Não Progressiva (ECINP), faixa etária de dois anos e cinco meses a 15 anos que compunham o grupo 2 (G2). Foram excluídos os adultos em fase de recuperação espontânea. Realizada análise retrospectiva dos protocolos de avaliação fonoaudiológica clínica com classificação do grau de comprometimento da disfagia, aplicados pré e pós - fonoterapia. Foi utilizado o Functional Oral Intake Scale - FOIS para avaliar o nível de ingestão oral, pré e pós-fonoterapia. Resultados. Quanto ao grau de comprometimento das disfagias houve mudança favorável somente nos adultos, sendo que na FOIS estas mudanças ocorreram nos dois grupos. Conclusáo. Houve mudanças favoráveis no grau de comprometimento da disfagia orofaríngea e nos níveis da FOIS, pré e pós - fonoterapia no AVC, porém na ECINP os marcadores utilizados não apresentaram mudanças favoráveis devendo inclusive ser revistos para aplicação nesta população. Serão necessários estudos futuros que investiguem as variáveis desta amostra.

Unitermos. Transtornos de Deglutição, Eficácia, Reabilitação, Neurologia.

Citaçáo. Pinto AR, Cola PC, Carvalho LR, Motonaga SM, Silva RG. Ingestáo Oral e Grau de Comprometimento da Disfagia Orofaríngea Neurogênica Pré e Pós-Fonoterapia.

\begin{abstract}
Objective. To compare, pre- and post-swallowing therapy, the level of oral intake scale, and the degree of severity of neurogenic oropharyngeal dysphagia. Method. 19 patients with oropharyngeal dysphagia: 10 Post-Stroke adults, aged from 44 years to 76 years (group 1 - G1), and nine children with Cerebral Palsy, aged from two years and five months to 15 years (group $2-\mathrm{G} 2$ ). We excluded individuals in the process of spontaneous recovery. We held retrospective analysis of clinical protocols for clinical speech therapy evaluation with classification of the degree of dysphagia severity, applied before and after swallowing therapy. We used the Functional Oral Intake Scale - FOIS to assess the level of oral ingestion, pre and post-swallowing therapy. Results. The degree of commitment of dysphagia was favorable change only in adults, and in FOIS these changes occurred in both groups. Conclusion. There were favorable changes in the degree of impairment of oropharyngeal dysphagia and levels of FOIS, pre and post - speech therapy in stroke, but in ECINP markers used showed no favorable changes should even be reviewed for application in this population. Future studies are needed to investigate the variables in this sample.
\end{abstract}

Keywords. Deglutition Disorders, Efficacy, Rehabilitation, Neurology.

Citation. Pinto AR, Cola PC, Carvalho LR, Motonaga SM, Silva RG. Oral Intake and Severity Scale In Neurogenic Oropharyngeal Dysphagia.

\footnotetext{
Trabalho realizado no Centro de Estudos da Educaçáo e da Saúde - CEES, UNESP - Campus Marília, Marília-SP, Brasil.

1.Fonoaudióloga, Mestranda do Programa de Pós-Graduação em Fonoaudiologia pela UNESP - Campus de Marília e Fonoaudióloga da Irmandade da Santa Casa de Misericórdia da cidade de Marília-SP, Brasil.

2.Fonoaudióloga, Doutora pela Faculdade de Medicina de Botucatu-UNESP, Docente do Curso de Medicina da Universidade de Marília e Assistente de Pesquisa do Laboratório de Disfagia da UNESP-Campus de Marília, Marília-SP, Brasil.

3.Bioestatística, Professora Assistente Doutor do Departamento de Bioestatística do Instituto de Biociências de Botucatu-UNESP, Botucatu-SP, Brasil.

4.Médica Otorrinolaringologista, Professora Assistente Doutor do Departamento de Fonoaudiologia da Universidade Estadual Paulista - UNESP e da Faculdade de Filosofia e Ciências/FCC - Campus de Marília, Marília-SP, Brasil. 5.Fonoaudióloga, Professora Assistente Doutor do Departamento de Fonoaudiologia da Universidade Estadual Paulista - UNESP e da Faculdade de Filosofia e Ciências/FCC - Campus de Marília, Marília-SP, Brasil.
}

Fonte de auxílio: Fundação de Amparo à Pesquisa do Estado de São Paulo - FAPESP; processo no 07/54352-4.

Endereço para correspondência: Roberta Gonçalves da Silva Avenida Higyno Muzzi Filho 737 CEP 17515-901, Marília-SP, Brasil. 


\section{INTRODUÇÃO}

As evidências sobre a eficácia da reabilitação na disfagia orofaríngea neurogênica têm sido foco atual de estudos na área, não havendo consenso quando se trata de distintas populaçóes disfágicas ${ }^{1,2}$.

A aplicação de técnicas terapêuticas baseadas no treinamento, ou na compensação da fisiologia da deglutição, durante o processo de reabilitação na disfagia orofaríngea, fez com que inicialmente os estudos se voltassem para a comprovação sobre a eficiência destas técnicas ${ }^{3}$. Além disto, a década de 90 é considerada um marco para as pesquisas sobre a definição de critérios para mensurar a eficácia da reabilitação em disfagia orofaríngea.

No início, as pesquisas sobre a reabilitação da disfagia orofaríngea se concentraram em estudar a eficiência das técnicas terapêuticas e apenas citar quais seriam os marcadores para mensurar a eficácia da reabilitação. Alguns estudos citavam que o objetivo desta reabilitação seria estabilizar o aspecto nutricional do indivíduo disfágico, eliminar os riscos de aspiração laringotraqueal e consequentes complicaçóes associadas ${ }^{4}$.

Mais recentemente distintos autores concordaram que a eficácia da reabilitação em disfagia orofaríngea deve ser avaliada mediante o impacto causado pela reabilitação em distintas variáveis, tais como nas alterações na fisiologia da deglutição, no nível de ingestão oral, nas manifestações pulmonares e nutricionais ${ }^{5,6}$.

Alguns destes estudos pesquisaram a eficácia da terapia na disfagia orofaríngea descrevendo programas de terapia para disfagia neurogênica crônica, salientando que as mudanças na fisiologia da deglutição direcionam as metas da terapia, mostrando benefícios funcionais duradouros e sem complicaçóes relacionadas à saúde durante o acompanhamento desses indivíduos ${ }^{7}$.

Com o objetivo de avaliar a eficácia das intervenções em crianças com comprometimento neurológico e disfagia orofaríngea, concluiu-se que havia necessidade de estudos randomizados que analisassem distintas variáveis e pudessem mensurar a eficácia das intervençôes para a disfagia orofaríngea ${ }^{8}$.

A partir de então, novos estudos vem relacionando as disfagias orofaríngeas e seus distintos diagnósticos etiológicos, mostrando que a duração da doença, o grau de aspiraçâo, o nível de ingestão oral do paciente, o nível cognitivo e outras variáveis influenciam de forma significante nos resultados da terapia de deglutiçãoo ${ }^{6,9,10}$.

A criação de escalas para aferir o grau de comprometimento da disfagia orofaríngea, bem como seu uso para facilitar as recomendaçôes quanto a melhor via para alimentação, ou para documentar a mudança no nível de ingestão oral funcional, são parâmetros que podem facilitar os protocolos propostos para mensurar também a eficácia da reabilitação ${ }^{11-15}$.

Outra questão sobre o controle de eficácia está relacionada à utilização de diferentes técnicas terapêuticas para o tratamento da disfagia orofaríngea em populaçôes distintas, sendo que a eficácia é maior para determinados grupos de pacientes, visto que a maioria dos estudos que investigaram as recomendaçôes e a eficácia da reabilitação não esclareceram a casuística estudada ${ }^{1}$.

Muitas são as doenças que apresentam disfagia como sintoma de base. O Acidente Vascular Cerebral (AVC) e a Encefalopatia Crônica Infantil não Progressiva (ECINP) caracterizam-se como duas patologias onde a incidência de disfagia orofaríngea é alta. A incidência de ECINP é de aproximadamente 2-2,5 por 1.000 nascidos vivos $^{16}$, e a prevalência de disfagia orofaríngea nesta população é estimada entre 19\% e 99\% ${ }^{17}$. Já no AVC a disfagia orofaríngea ocorre em mais de $50 \%$ dos pacientes e é uma das principais causas de mortalidade ${ }^{18}$. Além disso, poucos estudos compararam parâmetros específicos da reabilitação em indivíduos com disfagia pré e pós-fonoterapia. Considerando que a incidência e a prevalência de disfagia na população pós-AVC e com ECINP é alta, bem como as complicaçôes, torna-se necessário mensurar, nestes indivíduos, os resultados encontrados após fonoterapia.

Portanto, entendemos que a eficácia da reabilitação em disfagia orofaríngea está relacionada a modificaçôes no quadro clínico geral do indivíduo, seja no aspecto nutricional, no nível de ingestão oral, no controle de infecção pulmonar, no ganho nutricional e na importância da especificação da doença de base, associada à investigação do desempenho da biomecânica da deglutição.

Desta forma, o presente estudo teve por objetivo comparar o nível de ingestão oral e o grau de comprometimento na disfagia orofaríngea neurogênica pré e pós-fonoterapia. 


\section{MÉTODO}

\section{Sujeitos}

Trata-se de estudo clínico transversal retrospectivo, sendo selecionados 19 indivíduos com disfagia orofaríngea neurogênica, 15 do gênero masculino e quatro do gênero feminino, que participaram do Programa de Reabilitação no Laboratório de Disfagia de uma Instituição Pública no interior do Estado de São Paulo. Destes, 10 adultos Pós-Acidente Vascular Cerebral crônico (G1) com faixa etária variando de 44 a 76 anos, média 58,5 anos e nove crianças com Encefalopatia Crônica Infantil Náo Progressiva (G2) com faixa etária de dois anos e 5 meses a 15 anos, média 7,3 anos. Foram excluídos os adultos que estavam em fase de recuperação espontânea, ou que já haviam realizado algum programa de reabilitação para a disfagia.

O estudo foi analisado e aprovado pelo Comitê de Ética em Pesquisa de acordo com o processo $n^{\circ}$ 2866/2007.

\section{Procedimento}

O grau de comprometimento da disfagia orofaríngea foi coletado da avaliação fonoaudiológica clínica específica da deglutição, registrada pré e pós - fonoterapia. A avaliação específica da disfagia orofaríngea foi realizada por um fonoaudiólogo assistente de pesquisa com, no mínimo, dois anos de treinamento em disfagia. De acordo com o protocolo utilizado na Avaliação Fonoaudiológica da Disfagia Orofaríngea, a disfagia foi classificada como leve, moderada e grave ${ }^{15}$.

De acordo com o protocolo utilizado a disfagia orofaríngea foi classificada como leve, moderada ou grave. A disfagia leve (DL) é assim classificada pela alteração do esfíncter labial, incoordenação de língua, atraso para desencadear o reflexo de deglutição, ausência de tosse, sem redução acentuada da elevação da laringe, sem alteração da qualidade vocal após a deglutição e ausculta cervical sem alteração. A disfagia moderada (DM) ocorre devido à alteração do esfíncter labial, incoordenação de língua, atraso ou ausência do reflexo de deglutição, ausência de tosse, presença de tosse antes, durante ou após a deglutição, redução na elevação da laringe, alteração da qualidade vocal após a deglutição e ausculta cervical alterada. Já a disfagia grave (DG) dá-se pelo atraso ou ausência do reflexo de deglutição, redução na elevação da laringe, ausência de tosse, presença de tosse antes, durante ou após a deglutição, alteração da qualidade vocal após a deglutição, alteração respiratória evidente, deglutição incompleta e ausculta cervical alterada ${ }^{15}$.

Para a análise dos dados obtidos no decorrer desse estudo, quanto à mudança de grau de comprometimento na disfagia orofaríngea, foi considerado favorável a alteração de grave para moderado e de moderado para leve. A permanência da classificação em grau leve em determinados pacientes náo foi considerada.

Para avaliar o nível de ingestão oral, pré e pós - fonoterapia, os prontuários selecionados foram preenchidos com a Functional Oral Intake Scale (FOIS) composta por sete níveis ${ }^{14}$. Os níveis são: Nível 1: Nada por via oral; Nível 2: Dependente de via alternativa e mínima via oral de algum alimento ou líquido; Nível 3: Dependente de via alternativa com consistente via oral de alimento ou líquido; Nível 4: Via oral total de uma única consistência; Nível 5: Via oral total com múltiplas consistências, porém com necessidade de preparo especial ou compensaçôes; Nível 6: Via oral total com múltiplas consistências, sem necessidade de preparo especial ou compensações, porém com restriçôes alimentares; Nível 7: Via ora total sem restrições.

O programa de reabilitação aplicado neste serviço foi elaborado de forma individualizada e com a permanência obrigatória de um agente reabilitador para ambos os grupos. As terapias foram realizadas pelo profissional duas vezes por semana, e as orientaçôes foram sistemáticas para treinamento diário pelo agente reabilitador. Os pacientes receberam acompanhamento nutricional.

Para o G1 o programa constou de técnicas terapêuticas que incluíram manobras posturais de cabeça, modificação de consistências e volumes, algumas das manobras voluntárias de deglutição como de esforço, Mendelshon e deglutição múltipla, aplicação de estimulação térmica fria azeda ${ }^{19,20}$.

Para o G2 o programa constou de estratégias de posicionamento, controle de consistências e volume, controle na velocidade de oferta, mudanças de utensílios e técnicas de indução. 


\section{Análise Estatística}

Para comparação do grau de comprometimento entre os momentos pré e pós-fonoterapia, em cada grupo, foi utilizado o teste de Stuart-Maxwell para as variáveis leve, moderado e grave. $\mathrm{O}$ nível de significância utilizado foi de $5 \%$.

Para comparação da variável FOIS entre os momentos pré e pós-fonoterapia foi utilizado o teste $\mathrm{t}$ de Student em cada grupo. A distribuição foi normal nos 19 indivíduos pré e pós-fonoterapia.

\section{RESULTADOS}

Para analisar as mudanças ocorridas no grau de comprometimento pré e pós-fonoterapia, verificou-se que no G1 houve mudança favorável deste grau em três indivíduos (Tabela 1). No G2 não foram encontradas mudanças favoráveis no grau de comprometimento da disfagia, sendo que em 8 indivíduos o grau de comprometimento inclusive piorou (Tabela 2).

No G1 a FOIS variou entre os níveis (Tabela 3), sendo que após fonoterapia houve exclusão dos níveis que mencionavam via alternativa de alimentação, porém não houve diferença estatística. Já no G2, após fonoterapia, os níveis da FOIS, que incluíam via alternativa, aumentaram, verificando-se que houve diferença estatística $(\mathrm{p}<0,05)$.

\section{DISCUSSÃO}

$\mathrm{Na}$ década de 90 a incidência de disfagia orofaríngea foi estudada por vários pesquisadores, com o objetivo de determinar a prevalência deste sintoma nas etiologias neurológicas, tendo em vista a necessidade de elegermos planejamentos terapêuticos apropriados para as distintas populaçóes disfágicas ${ }^{21}$.

As dificuldades encontradas nas pesquisas sobre a eficácia da reabilitação em disfagia orofaríngea foi recentemente destacada pelos estudos de revisão. Uma das questôes mais marcantes desta discussão é a heterogeneidade das amostras estudadas, o que, portanto, tem contribuído para dificultar a compreensão sobre as variáveis de cada população no contexto da reabilitação ${ }^{2,3}$. Esta abordagem metodológica, que chegou inclusive a incluir disfagias mecânicas e neurogênicas no mesmo estudo, atrasou nossa compreensão sobre como os diferentes
Tabela 1

Distribuição de frequências dos individuos (G1) segundo o grau de comprometimento da disfagia orofaringea pré e pós-fonoterapia

\begin{tabular}{|c|c|c|c|}
\hline & & Pré-fonoterapia & Pós-fonoterapia \\
\hline \multirow{3}{*}{ Disfagia } & Grave & 2 & 1 \\
\hline & Moderada & 5 & 4 \\
\hline & Leve & 3 & 5 \\
\hline \multicolumn{2}{|l|}{ TOTAL } & 10 & 10 \\
\hline
\end{tabular}

$\mathrm{P}=0,22$ (teste de Stuart-Maxwell)

Tabela 2

Distribuição de frequências dos individuos (G2) segundo grau de comprometimento da disfagia orofaringea pré e pós-fonoterapia

\begin{tabular}{|c|c|c|c|}
\hline & & Pré-fonoterapia & Pós-fonoterapia \\
\hline \multirow{3}{*}{ Disfagia } & Grave & 3 & 6 \\
\hline & Moderada & 4 & 3 \\
\hline & Leve & 2 & 0 \\
\hline \multicolumn{2}{|l|}{ TOTAL } & 9 & 9 \\
\hline
\end{tabular}

$\mathrm{P}=0,08$ (teste de Stuart-Maxwell)

Tabela 3

Média referentes à FOIS segundo os grupos pré e pós-fonoterapia

\begin{tabular}{cccc}
\hline & \multicolumn{3}{c}{ Momento } \\
\cline { 2 - 3 } & Pré -Fonoterapia & Pós -Fonoterapia & \\
\hline \multirow{2}{*}{ G1 } & 5,8 & 5,6 & $\mathrm{p}>0,05$ \\
& 2,1 & 1,1 & \\
G2 & 5,2 & 4,2 & $\mathrm{p}<0,05$ \\
& 1,3 & 1,3 & \\
\hline
\end{tabular}

$\mathrm{p}=0,54$ no G1; $\mathrm{p}=0,04$ no $\mathrm{G} 2$ (teste $\mathrm{t}$ de Student)

comprometimentos na biomecânica da deglutição reagem ao processo de reabilitação na disfagia orofaríngea. Assim, as diversas críticas sobre esta metodologia fizeram com que, mais recentemente, as pesquisas procurassem delinear estudos com grupos mais homogêneos, inclusive com casos únicos ${ }^{10,22}$.

Selecionar parâmetros para controlar as mudanças ocorridas após o processo terapêutico na disfagia orofaríngea ainda é um procedimento em evolução nas pesquisas nesta área ${ }^{10}$. A aplicação do grau de comprometimento da disfagia, pré e pós-fonoterapia é uma das muitas possibilidades para compreender o desempenho da biomecânica da deglutição neste processo. Entendemos, no entanto, 
que a maioria das escalas propostas para este fim prioriza a presença de penetração laríngea e aspiração laringotraqueal como o mais importante marcador de risco $^{10}$ e não incluem as variaçóes do tempo de trânsito oral (TTO) que podem comprometer o estado nutricional. Esta questão é de fundamental relevância no Acidente Vascular Cerebral (AVC) e na Encefalopatia Crônica Infantil Não Progressiva (ECINP), uma vez que dependendo do comprometimento não há aspiração laringotraqueal, mas o TTO é muito aumentado e dificulta a presença da via oral total.

Dados da literatura atual ressaltam a gravidade da condição neurológica e a manifestação da disfagia, levando-se em consideração os achados da avaliação clínica fonoaudiológica, favorecendo um melhor entendimento sobre a biomecânica da deglutição ${ }^{23}$.

Assim, a presença de mudanças favoráveis no grau de comprometimento ou a permanência de indivíduos em grau leve encontrada em nossos resultados no G1, após fonoterapia, comprova que o impacto da reabilitação para a biomecânica da deglutição no AVC pode ser conquistado com fonoterapia, assim como já descrito por alguns autores ${ }^{10,24}$. Devemos ressaltar, no entanto, que as lesôes encefálicas na população de AVC comprometem em maior ou menor grau a deglutição orofaríngea ${ }^{12}$, sendo que neste momento nosso estudo não é capaz de esclarecer estas relaçôes. Há, no entanto, relatos na literatura que referem que as lesóes de hemisfério cerebral direito e tronco encefálico provocam disfagias mais graves do que as encontradas em hemisfério cerebral esquerdo ${ }^{25}$.

A eficácia da reabilitação na disfagia orofaríngea na ECINP é pouco descrita na literatura, sendo que um dos trabalhos realizados com esta população verificou que as diferentes abordagens terapêuticas envolvendo a deglutição não são capazes de garantir suporte nutricional satisfatório por via oral ${ }^{26}$. No entanto, deve-se ressaltar que há dificuldade em mensurar resultados nesta populaçâo devido às distintas complicaçóes que acompanham esta patologia e que tanto colocam a saúde desta população em constante instabilidade. Portanto, a ausência de mudanças favoráveis no grau de comprometimento da disfagia pós - fonoterapia no G1 pode estar relacionada tanto ao tipo de escala usada neste estudo, como com as co-morbidades que afetam esta população.

Portanto, torna-se necessário a discussão sobre quais seriam os parâmetros mais adequados para mensurar a intervenção fonoaudiológica na ECINP. Uma vez que a fonoterapia não modificou favoravelmente o desempenho da biomecânica da deglutição nem o nível de ingestão oral, sugere-se que as complicaçôes, sejam estas de origem orgânica ou intelectual encontradas nesta população, sejam discutidas como marcadores deste controle de eficácia ${ }^{27,28}$.

Conforme já apontado na literatura a FOIS parece ser um importante parâmetro para mensurar o nível de ingestão oral pré e pós - fonoterapia na disfagia orofaríngea apontando as mudanças ocorridas na alimentação durante o processo terapêutico na disfagia orofaríngea ${ }^{10,18,29}$.

A presença de mudanças favoráveis na FOIS encontradas no G1 e a ausência no G2 esta possivelmente relacionada ao fato deste segundo grupo caracterizar-se por uma população que chega ao atendimento fonoterápico com dieta via oral não gerenciada, quando muitas vezes deveria estar em uso de via alternativa de alimentação. Assim, a FOIS pré-fonoterapia está em nível alto na escala, acabando por sofrer rebaixamento como parte da orientação do programa de reabilitação a fim de prevenir complicaçôes.

Portanto, entendemos que ainda é necessária uma ampla discussão sobre os marcadores que devem ser incluídos nos protocolos para controle de eficácia terapêutica dependendo da população estudada, podendo ser esta a chave da comprovação da eficácia na reabilitação da disfagia orofaríngea.

\section{CONCLUSÃO}

Há mudanças favoráveis no grau de comprometimento da disfagia orofaríngea e nos níveis da FOIS, pré e pós-fonoterapia no AVC, porém na ECINP os marcadores utilizados não apresentaram mudanças favoráveis devendo inclusive ser revistos para aplicação nesta população. 


\section{REFERÊNCIAS}

1.Logemann JA. Oropharyngeal dysphagia and nutritional management. Curr Opin Clin Nutr Metab Care 2007;10(5):611-4.

2.Coyle J, Davis L, Easterling C, Graner D, Langmore S, Leder S, et al. Oropharyngeal dysphagia assessment and treatment eficacy: setting the record straight. J Am Med Dir Assoc 2009;10:62-6.

http://dx.doi.org/10.1016/j.jamda.2008.10.003

3.Silva RG. A eficácia da reabilitação em disfagia orofaríngea. Revista Pró-Fono 2007;19(1):123-30.

4.Depippo KL, Holas MA, Reding MJ, Mandel FS, Lesser ML. Dysphagia therapy following stroke: a controlled trial. Neurology 1994;44(9):1655-60.

http://dx.doi.org/10.1212/WNL.44.9.1655

5.Langmore SE. Efficacy of behavioral treatment for oropharyngeal dysphagia. Dysphagia 1995;10(4):259-62.

http://dx.doi.org/10.1007/BF00431419

6.Logemann JR. Treatment of oral and pharyngeal dysphagia. Phys Med Rehabil Clin N Am 2008;19(4):803-16.

http://dx.doi.org/10.1016/j.pmr.2008.06.003

7.Carnaby-mann GD, Crary MA. Adjuntive neuromuscular electrical stimulation for treatment-refractory dysphagia. Ann Otol Rhinol Laryngol. 2008;117(4):279-87.

8.Morgan AT, Dodrill P, Ward EC. Interventions for oropharyngeal dysphagia in children with neurological impairment. Cochrane Database Syst Rev 2012 Oct 17;10:CD009456.

9.Furkim AM, Sacco ABF. Eficácia da fonoterapia em disfagia neurogênica usando a escala funcional de ingestâo por via oral (FOIS) como marcador. Rev CEFAC 2008;10(4):503-12.

http://dx.doi.org/10.1590/S1516-18462008000400010

10.Silva RG, Jorge AG, Peres FM, Cola PC, Gatto AR, Spadotto AA. Protocolo para Controle de Eficácia Terapêutica em Disfagia Orofaríngea Neurogênica (Procedon). Rev CEFAC 2010;12:75-81. http://dx.doi.org/10.1590/S1516-18462010000100010

11.Ott D, Hodney R, Pikna LA, Chen M, Gelfand D. Modified barium swallow: clinical and radiographic correlation and relation to feeding recommendations. Dysphagia 1996;11:187-90.

http://dx.doi.org/10.1007/BF00366383

12.Daniels S, Mcadam C, Brailey K, Foundas A. Clinical assessment of swallowing and prediction of dysphagia severity. Am J Speech Lang Pathol 1997;6:17-24.

13.O' Neil KH, Purdy M, Falk J, Gallo L. The dysphagia outcome and severity scale. Dysphagia 1999;14(3):139-45.

http://dx.doi.org/10.1007/PL00009595

14.Crary MA, Mann GD, Groher ME. Initial psychometric assessment of a functional oral intake scale for dysphagia in stroke patients. Arch Phys Med Rehabil 2005;86(8):1516-20.

http://dx.doi.org/10.1016/j.apmr.2004.11.049

15.Silva RG. Disfagia Orofaríngea Pós-acidente Vascular Encefálico. In: Ferreira LP, Belfi-Lopes DM, Limonge SCO. (Orgs.) Tratado de Fonoaudiologia. São Paulo: Roca, 2004, p.354-69.
16.Erasmus CE, van Hulst K, Rotteveel JJ, Willemsen MA, Jongerius PH. Clinical practice: swallowing problems in cerebral palsy. Eur J Pediatr 2012;171(3):409-14.

http://dx.doi.org/10.1007/s00431-011-1570-y

17.Benfer KA, Weir KA, Bell KL, Ware RS, Davies PS, Boyd RN. Longitudinal cohort protocol study of oropharyngeal dysphagia: relationships to gross motor attainment, growth and nutritional status in preschool children with cerebral palsy. BMJ Open 2012;13:2(4).

18. Rofes L, Vilardell N, Clavé P. Post-stroke dysphagia: progress at last. Neurogastroenterol Motil 2013;25(4):278-82.

http://dx.doi.org/10.1111/nmo.12112

19.Logemann JA, Pauloski BR, Colangelo L, Lazarus C, Fujiu M, Kahrikas PJ. Effects of a sour bolus on oropharyngeal swallowing measures in patients with neurogenic dysphagia. J Speech Hear Res 1995;38(3):556-63.

20.Cola PC, Gatto AR, Silva RG, Spadotto AA, Schelp AO, Henry MACA. The influence of sour taste and cold temperature in pharyngeal transit duration in patients with stroke. Arquivos de Gastroenterologia 2010;47:18-21.

21.Furkim AM, Behlau MS, Weckx LLM. Avaliação Clínica e Videofluoroscopica da Deglutição em Crianças com Paralisia Cerebral Tetraparética Espástica. Arq Neuropsiquiatr 2003;61(3-A):611-6.

http://dx.doi.org/10.1590/S0004-282X2003000400016

22.Bedgood R, Bullock MA. A case of occult oropharyngeal dysphagia. South Med J 2006;99(5):548.

http://dx.doi.org/10.1097/01.smj.0000216426.73236.d0

23.Itaquy RB, Favero SR, Ribeiro Mde C, Barea LM, Almeida ST, Mancopes R. Dysphagia and cerebrovascular accident: relationship between severity degree and level of neurological impairment. J Soc Bras Fonoaudiol 2011;23(4):385-9.

http://dx.doi.org/10.1590/S2179-64912011000400016

24.Remesso GC, Fukujima MM, Chiappetta AL, Oda AL, Aguiar AS, Oliveira Ade S, do Prado GF. Swallowing disorders after ischemic stroke. Arq Neuropsiquiatr 2011;69(5):785-9.

http://dx.doi.org/10.1590/S0004-282X2011000600012

25.Hamdy S, Mikulis DJ, Crawley A, Xue S, Lau H, Henry S et al. Cortical activation during human volational swallowing: an event-related fMRI study. Am J Physiol. 1999;277(1pt 1):G219-25.

26.Gisel EG. Oral-motor skills following sensorimotor intervention in the moderately eating-impaired child with cerebral palsy. Dysphagia 1994;9(3):180-92. http://dx.doi.org/10.1007/BF00341263

27.Henderson CM, Rosasco M, Robinson LM, Meccarello J, Janicki MP, Turk MA, et al. Functional impairment severity is associated with health status among older persons with intellectual disability and cerebral palsy. J Intellect Disabil Res 2009;53(11):887-97.

http://dx.doi.org/10.1111/j.1365-2788.2009.01199.x

28.Pruitt DW, Tsai T. Common medical comorbidities associated with cerebral palsy. Phys Med Rehabil Clin N Am 2009;20(3):453-67.

http://dx.doi.org/10.1016/j.pmr.2009.06.002

29. Carnaby-Mann GD, Crary MA. McNeill dysphagia therapy program: a case-control study. Arch Phys Med Rehabil 2010;91(5):743-9.

http://dx.doi.org/10.1016/j.apmr.2010.01.013 